\title{
Chandra-LETGS X-ray observation of $\alpha$ Centauri: A nearby (G2V + K1V) binary system
}

\author{
A. J. J. Raassen ${ }^{1,2}$, J.-U. Ness ${ }^{3}$, R. Mewe ${ }^{1}$, R. L. J. van der Meer ${ }^{1}$, V. Burwitz ${ }^{4}$, and J. S. Kaastra ${ }^{1}$ \\ 1 SRON National Institute for Space Research, Sorbonnelaan 2, 3584 CA Utrecht, The Netherlands \\ 2 Astronomical Institute "Anton Pannekoek", Kruislaan 403, 1098 SJ Amsterdam, The Netherlands \\ 3 Universität Hamburg, Gojenbergsweg 112, 21029 Hamburg, Germany \\ ${ }^{4}$ Max-Planck-Institut für extraterrestrische Physik, Postfach 1312, 85741 Garching, Germany
}

Received 7 October 2002 / Accepted 23 December 2002

\begin{abstract}
A Chandra LETGS X-ray observation of $\alpha$ Centauri with an exposure time of $81.5 \mathrm{ks}$ is presented with the two components (K1V and G2V) spectrally resolved for the first time. We use the emission lines from the individual spectra to determine plasma temperatures and find similar temperatures as for the Sun with higher temperatures for the K1V star than for the G2V star. Global fitting techniques are used in order to construct an emission measure distribution for each star and we find emission measure distributions consistent with what is found from the line ratios. A two-temperature model is used in order to derive abundances normalized to iron and relative to solar photosheric values. For both stars we find a FIP effect with a slight but not significant tendency of a stronger FIP effect for the K1V component.
\end{abstract}

Key words. stars: individual: $\alpha$ Centauri - stars: coronae - stars: late-type - stars: activity - X-rays: stars

\section{Introduction and observation}

Late-type F-M stars with photospheric temperatures between 4000 and $7000 \mathrm{~K}$ show hot outer atmospheres (coronae) (Schmitt 1997) with temperatures around 1-10 MK. Due to the high temperature these plasmas emit X-ray radiation. This steep rise of the temperature above the stellar surface by 3 orders of magnitude is - after decades of investigations - still a puzzling problem and not well understood. The solar corona shows rich details on structures, mass motions, abundance patterns, loops and flares. These phenomena are driven by magnetic activity.

From studies by, e.g., Güdel et al. (1997a,b) of a series of solar-type stars of different ages with ROSAT, EUVE, and ASCA, it is established that the relatively old, non-flaring Sun has a rather inactive corona with temperatures in the range 1$3 \mathrm{MK}$, while the more active (and younger) G dwarfs like EK Dra have coronae with both $\sim 5$ and $\gtrsim 10 \mathrm{MK}$ plasmas. The latter temperature is reached in the solar corona only during short flares.

However, many of the stellar objects, regularly observed by X-ray satellites belong to the more abundant low-mass stars ( $\mathrm{M}$ and $\mathrm{K}$ dwarfs) with high $\mathrm{X}$-ray luminosities and flares and therefore very high magnetic activity. This magnetic activity has been found to be correlated with the rotational velocity on the stellar surface (e.g., Pallavicini et al. 1981;

Send offprint requests to: A. J. J. Raassen,

e-mail: A.J.J.Raassen@sron.nl
Güdel et al. 1997b) and a dynamo process is the most plausible source of magnetic field generation in these stars.

The components $\mathrm{A}$ and $\mathrm{B}$ of the binary $\alpha$ Centauri (HD 128620;1) are slightly older than the Sun and are therefore expected to have a coronal activity comparable to the solar corona (cf. e.g., Güdel et al. 1997b). The binary system $\alpha$ Centauri is the nearest stellar system at a distance of 1.34 pc. It consists of a $1.1 M_{\odot} \mathrm{G} 2 \mathrm{~V}$ star with a radius of $1.24 R_{\odot}$ and a $0.9 M_{\odot} \mathrm{K} 1 \mathrm{~V}$ star with a radius of $0.84 R_{\odot}$ (e.g., Flannery \& Ayres 1978). The orbit of the binary system is wide (semi-major axis $23.5 \mathrm{AU}=17.5^{\prime \prime}$ ), with a period of 80.1 years (Flannery \& Ayres 1978). The rotational periods of A and B are 29 and 42 days (Hallam et al. 1991; Saar \& Osten 1997) and the corresponding rotational velocities $2.7 \pm 0.9$ and $1.1 \pm 0.8 \mathrm{~km} \mathrm{~s}^{-1}$, respectively (Saar \& Osten 1997). According to the X-ray luminosity-velocity relations given by Pallavicini et al. (1981) and Güdel et al. (1997b) the velocities characterize the corona $\alpha$ Cen as rather inactive (like the solar corona).

The system has been studied in X-rays in the past, e.g, by Einstein (Golub et al. 1982), EUVE (Drake et al. 1997, Mewe et al. 1995b), ASCA (Mewe et al. 1998a), BeppoSAX (Mewe et al. 1998b), and ROSAT (Schmitt 1997 and 1998). Though the ROSAT and Einstein HRI detectors have separated the two components to measure fluxes, spectra of the two individual stars could never be obtained separately.

After the launch of Chandra and XMM-Newton the $\alpha$ Centauri binary system can be studied in more detail in the X-ray region. The spectra of the G2V and K1V star 

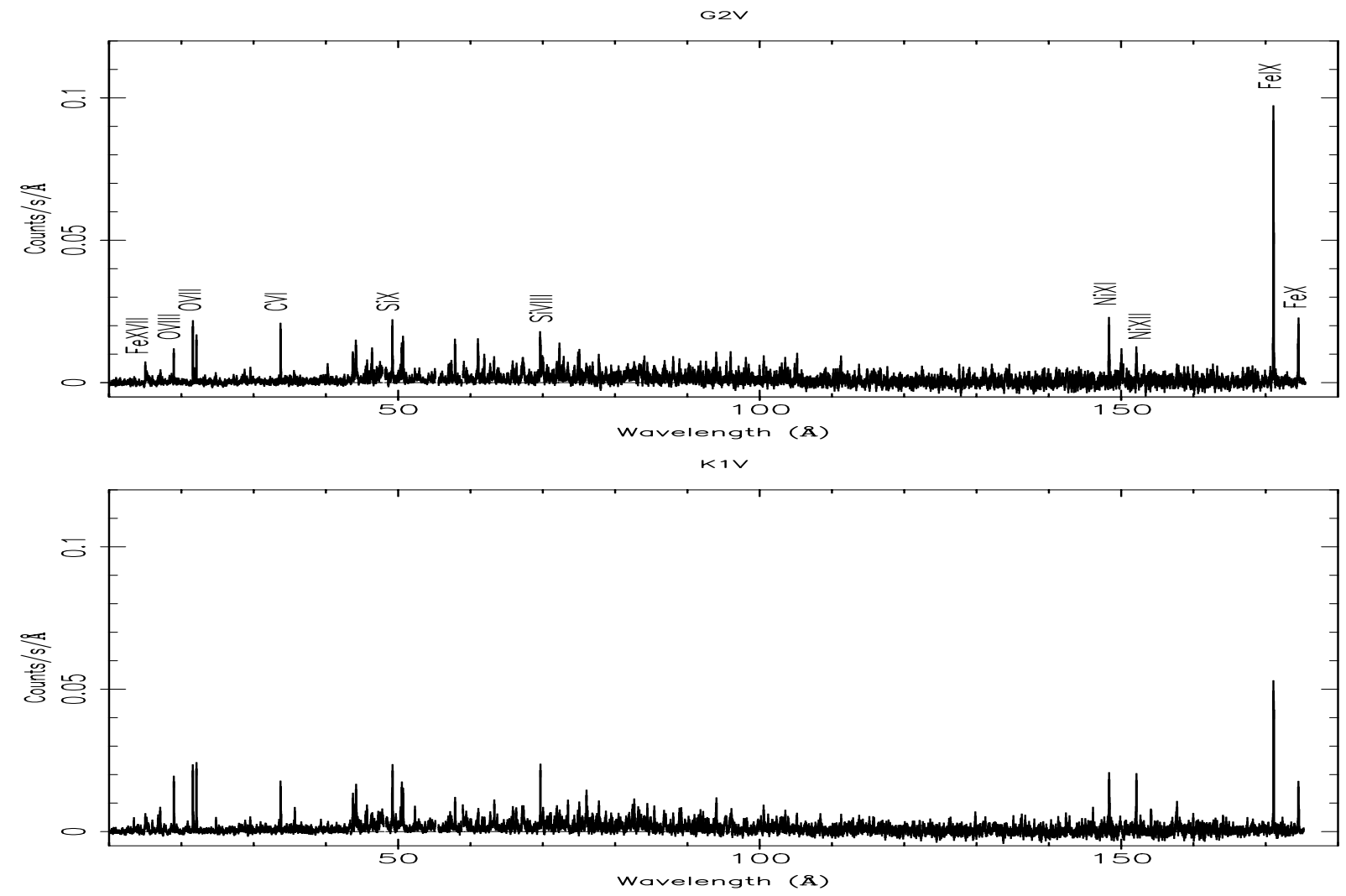

Fig. 1. The spectra of the binary system $\alpha$ Centauri in the range from 10 to $180 \AA$, obtained by LETGs. On top G2V star, below K1V.

were obtained (on December 25 1999) with an exposure time of $81.5 \mathrm{ks}$ by the Low Energy Transmission Grating (LETG) in combination with the High Resolution Camera (HRC-s) on board Chandra. During that observation the two stars were separated by $16^{\prime \prime}$ on the sky. The dispersion axis was positioned nearly perpendicular to the axis of the binary, resulting in two separated spectra. This offers the possibility to study the differences and similarities between the two stars in detail. To obtain the spectra from the Chandra data we used the standard CXC pipeline products, later updated with the standard CIAO reduction: CIAO 2.2 with the science threads for LETG/HRC-S observations. We then used our own software based on FTOOLS routines to separate the two spectra. We used the standard 'bow-tie-shaped' extraction box for the spectrum of each star. The width of the extration box at the longest wavelength is less than $10^{\prime \prime}$. Since the spectra had a separation of $16^{\prime \prime}$, the extraction boxes do not overlap. To avoid having the spectrum of the other star in the background, we used a background box of $30^{\prime \prime}$ on only one side of the star (opposite of the other component). This lowers the statistics of the background, but the background box is still sufficiently large.

For the analysis the spectra of the +1 and -1 order were summed. The spectra are background-subtracted. For the effective area we have used the values as calibrated in flight at SRON (van der Meer et al. 2003), which agree within about 5$10 \%$ with the values, as given in the Chandra LETGs Calibration Review of 31 Oct. $2001^{1}$.

\footnotetext{
${ }^{1}$ http://cxc.harvard.edu/cal/Links/Letg/User/Review_ 311001/ eff_area.html
}

Figure 1 shows the spectra of $\alpha$ Cen G2V (top) and K1V (bottom) obtained by LETGS in the range from 10 to $180 \AA$. The effective area calibration at long wavelength is based on the spectra of HZ43 and Sirius B. The error on the effective area at $170-175 \AA$ is about $10-15 \%$.

\section{Analysis}

\subsection{Individual lines and individual line fitting}

The lines in the spectrum have been modeled locally using a narrow Gaussian line profile, which was folded with the instrumental Line-Spread-Function (LSF), together with a constant representing the background and (pseudo)continuum. The average instrumental $F W H M$ of the lines is about $0.06 \AA$. The fluxes have been obtained after correction for the effective area. The statistical errors in the line fluxes include background errors. Table A.1 shows the measured wavelengths and line fluxes (not corrected for interstellar absorption), together with identifications obtained from Kelly (1987).

In Fig. 1 we recognize the dominant $\mathrm{Fe}$ IX and $\mathrm{Fe} x$ lines between 170 and $175 \AA$ (which are even more prominent when corrections for the relatively low effective area are made) and strong $\mathrm{H}$-like and He-like lines of $\mathrm{C}, \mathrm{N}$, and $\mathrm{O}$ in the wavelength region below $40 \AA$. The latter are better seen in Fig. 2 covering the wavelength range from 10 to $45 \AA$. From Fig. 1 we notice that the intensity of the "cool" Fe Ix line relative to the "hot" O VIII line at $18.969 \AA$ is higher for the G2V star (top panel) than for the K1V star. A similar temperature behaviour is seen from Fig. 2 with respect to the "cool" C v and C vi lines which are higher relative to the "hotter" $\mathrm{O}$ VII and $\mathrm{O}$ VIII lines in 

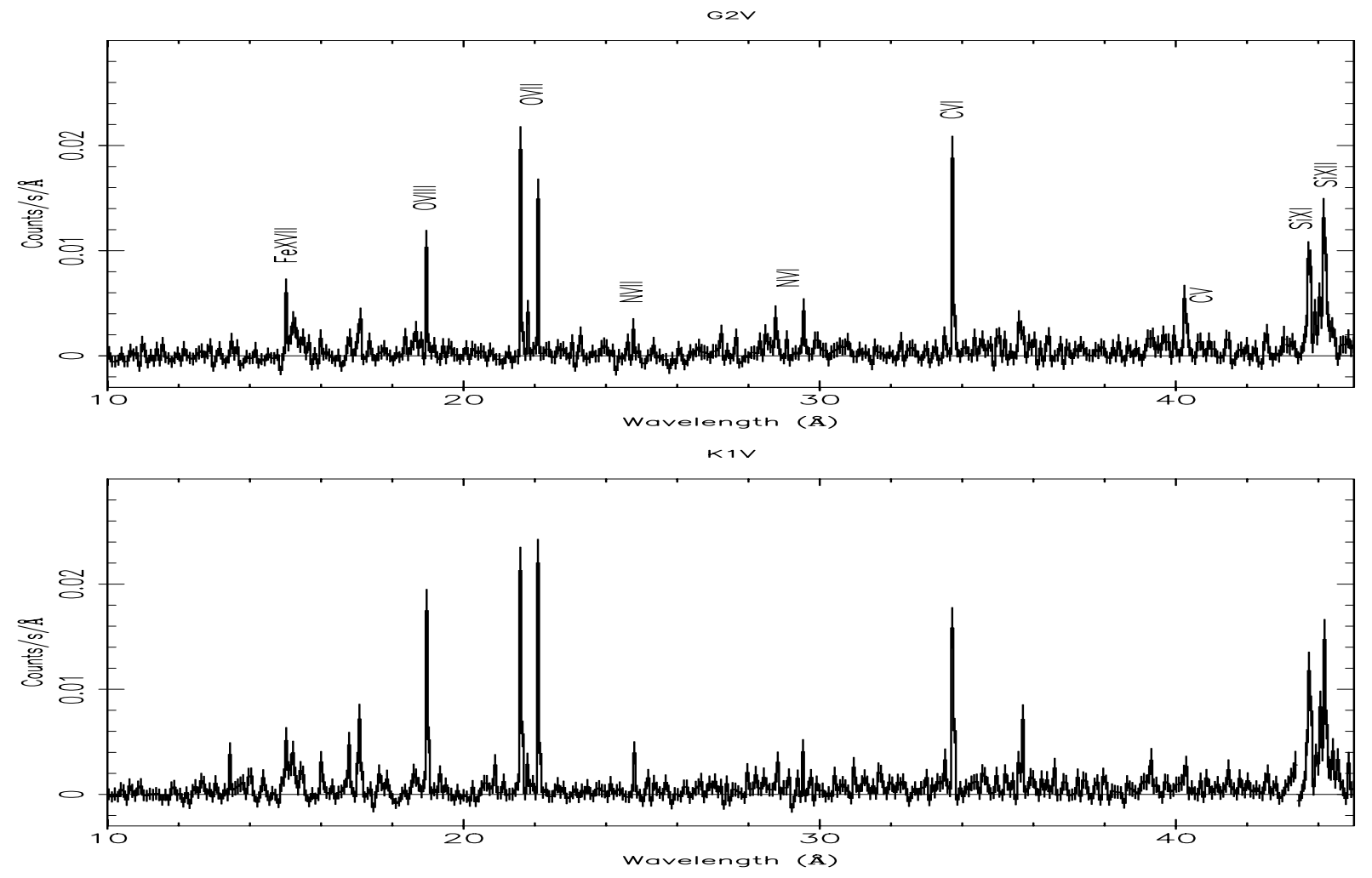

Fig. 2. Spectra of $\alpha$ Centauri G2V and K1V in the wavelength range from 10 to $45 \AA$.

Table 1. Line flux ratios between lines of the G2V and K1V star for different ions in order of increasing peak formation temperature $T_{\mathrm{m}}$.

\begin{tabular}{lrcl}
\hline \hline ion & $\lambda(\AA)$ & G2V/K1V & $T_{\mathrm{m}}(\mathrm{MK})$ \\
\hline Fe IX & 171.075 & 1.54 & 0.71 \\
Fe X & 174.534 & 1.04 & 0.95 \\
Fe XI & 89.185 & 0.43 & 1.3 \\
Fe XI & 86.772 & 0.55 & 1.3 \\
C V(r) & 40.268 & 1.71 & 0.93 \\
C VI(r) & 33.736 & 0.99 & 1.4 \\
N VI(r) & 28.787 & 1.04 & 1.4 \\
N VII(r) & 24.781 & 0.50 & 2.1 \\
O VII(r) & 21.602 & 0.77 & 2.1 \\
O VIII(r) & 18.969 & 0.51 & 3.3 \\
\hline
\end{tabular}

the spectrum of the G2V star than in the spectrum of the $\mathrm{K} 1 \mathrm{~V}$ star. This trend is shown in Table 1 . In that table we give the calculated line flux ratios between $\mathrm{G} 2 \mathrm{~V}$ and $\mathrm{K} 1 \mathrm{~V}$, together with the optimal line formation temperature. In the lower temperature regime the lines are stronger in the spectrum of the $\mathrm{G} 2 \mathrm{~V}$ star than in the spectrum of the K1V star, while for "hotter" lines the lines in the G2V star have lower fluxes than those in the K1V star. This trend is illustrated in Fig. 3 in which the line flux ratios $(\mathrm{G} 2 \mathrm{~V} / \mathrm{K} 1 \mathrm{~V})$ of a number of Si-ions and $\mathrm{Fe}$-ions are shown relative to the temperature of optimum line formation. It is seen that the line flux ratio between the G2V and the $\mathrm{K} 1 \mathrm{~V}$ star is decreasing with the temperature. The shift between the silicon and iron ratios reflects the abundance difference between the two stars (see also Table 3 ). Table 1 shows that
Table 2. Temperature and electron density dependent line ratios in $\mathrm{C}$, $\mathrm{N}$, and $\mathrm{O}$, together with the derived temperature.

\begin{tabular}{lllll}
\hline \hline & \multicolumn{2}{c}{$\alpha$ Cen $(\mathrm{G} 2 \mathrm{~V})$} & \multicolumn{2}{c}{$\alpha$ Cen $(\mathrm{K} 1 \mathrm{~V})$} \\
\multicolumn{1}{c}{$T_{\text {rad }}(\mathrm{K})^{a}$} & \multicolumn{2}{c}{4700} & \multicolumn{2}{c}{4100} \\
\hline lines & ratio & $T(\mathrm{MK})$ & ratio & $T(\mathrm{MK})$ \\
O VII(i+f)/O VII(r) & $1.00(0.17)$ & $1.5(0.5)$ & $1.07(0.16)$ & $1.5(0.5)$ \\
O VII(r)/O VIII(r) & $3.11(0.68)$ & $1.8(0.1)$ & $2.06(0.34)$ & $2.0(0.1)$ \\
N VI(r)/N VII(r) & $2.40(1.13)$ & $1.3(0.2)$ & $1.15(0.46)$ & $1.5(0.2)$ \\
C V(r)/C VI(r) & $0.64(0.12)$ & $1.1(0.1)$ & $0.37(0.09)$ & $1.3(0.1)$ \\
lines & ratio & density $^{b}$ & ratio & density \\
O VII(f/i) & $3.7(1.1)$ & $<10^{10}$ & $6.8(2.4)$ & - \\
$\mathrm{N} \mathrm{VI(f/i)}$ & $4.0(2.0)$ & $<8 \times 10^{9}$ & $2.9(2.3)$ & $<4 \times 10^{10}$ \\
$\mathrm{C} \mathrm{V}(\mathrm{f} / \mathrm{i})$ & $2.7(1.0)$ & $<2 \times 10^{9}$ & $3.8(1.7)$ & $0.5-3 \times 10^{9}$ \\
\hline
\end{tabular}

${ }^{a}$ From Ness et al. (2002).

${ }^{b}$ From Porquet et al. (2001) and in units of $\mathrm{cm}^{-3}$.

flux ratios from other elements $(\mathrm{C}, \mathrm{N}, \mathrm{O})$ are in line with the Si-ratios.

A second temperature indication for the two stars is obtained from line flux ratios between the "cooler" He-like lines of C v, N vI, and $\mathrm{O}$ vII and the "hotter" H-like resonance lines of $\mathrm{C}$ VI, $\mathrm{N}$ VII, and $\mathrm{O}$ VIII.

In addition, the three O VII lines themselves are densityand temperature-dependent. The "triplet" consists of the $1 \mathrm{~s}^{2}{ }^{1} \mathrm{~S}_{0}-1 \mathrm{~s} 2 \mathrm{p}{ }^{1} \mathrm{P}_{1}$ resonance line $(r)$, the $1 \mathrm{~s}^{2}{ }^{1} \mathrm{~S}_{0}-1 \mathrm{~s} 2 \mathrm{p}^{3} \mathrm{P}_{1}$ intercombination line $(i)$, and the $1 \mathrm{~s}^{2}{ }^{1} \mathrm{~S}_{0}-1 \mathrm{~s} 2 \mathrm{~s}{ }^{3} \mathrm{~S}_{1}$ forbidden line $(f)$. The $1 \mathrm{~s} 2 \mathrm{~s}{ }^{3} \mathrm{~S}_{1}$ level is metastable and can only decay to 


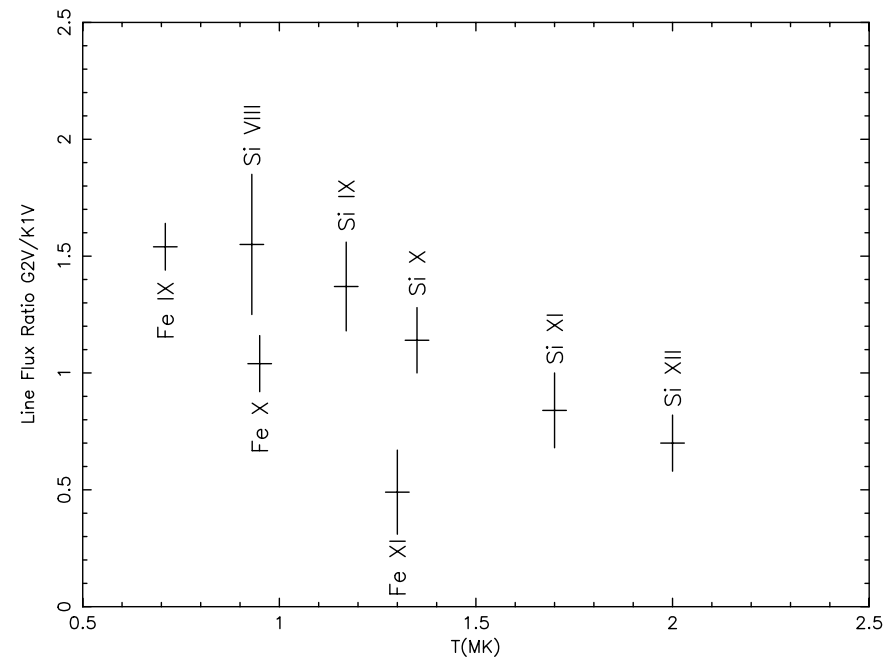

Fig. 3. The ratio between the line fluxes of the G2V star and the K1V star compared to the temperature of optimal line formation for five silicon ions and three iron ions.

the ground by means of a forbidden magnetic dipole transition. However, the level will be depopulated by electron collisions at higher densities in favour of the $1 \mathrm{~s} 2 \mathrm{p}^{3} \mathrm{P}_{1}$ level. This results in a decrease of the forbidden line and an increase of the intercombination line. Therefore the value $R=f / i$ is an adequate diagnostic tool to constrain the density. The sum of the intercombination line and the forbidden line relative to the resonance line, $G=(i+f) / r$ is a temperature indicator. Results of this comparison of the temperatures of the $\mathrm{G} 2 \mathrm{~V}$ and the $\mathrm{K} 1 \mathrm{~V}$ star are given in Table 2, showing higher ratios for the "cooler" (He-like) ions in the G2V star. For completeness the density-sensitive Helike $(f / i)$ lines ratios have been inserted in Table 2 . The values agree with Ness et al. (2002). For density diagnostics we refer to that paper.

The analysis of the individual line fluxes of the two stars leads to the conclusion that the corona of the K1V star is hotter than that of the G2V star.

\subsection{Global fitting}

In addition to the analysis based on the individual line fluxes we characterize the thermal structure and the elemental composition of both coronae (K1V and G2V) by means of global fitting to the spectra as a whole. To this end, we fitted multi- $T$ models using SPEX (Kaastra et al. 1996a) in combination with the MEKAL database (Mewe et al. 1985; Mewe et al. 1995a) of which an extended line list can be found on the web ${ }^{2}$. For the electron density $n_{\mathrm{e}}$ we assumed the values given in Table 2 and the interstellar column density $N_{\mathrm{H}}$ is taken equal to the value in Table 3. For both coronal plasmas the calculations require two dominant temperature components. The reduced $\chi^{2}$ is relatively high (1.4) for the fits. This is due to a lack of lines in the

\footnotetext{
${ }^{2}$ http://www.sron.nl/divisions/hea/spex/ version $1.10 /$ line/
}

Table 3. Multi-temperature fitting and $D E M$ modeling for the K1V and G2V stars. Elemental abundances for the three instruments are given normalized to iron and relative to solar photospheric values given by Anders \& Grevesse (1989), except for $\mathrm{Fe}^{a} .1 \sigma$ uncertainties are given in brackets.

\begin{tabular}{|c|c|c|}
\hline \multirow[b]{2}{*}{ Parameter } & \multicolumn{2}{|c|}{ Multi- $T$ fitting } \\
\hline & $\mathrm{K} 1 \mathrm{~V}$ & $\mathrm{G} 2 \mathrm{~V}$ \\
\hline $\log N_{\mathrm{H}}\left[\mathrm{cm}^{-2}\right]^{b}$ & 17.95 & 17.95 \\
\hline$T_{1}[\mathrm{MK}]$ & $1.17(2)$ & $1.10(2)$ \\
\hline$T_{2}[\mathrm{MK}]$ & $2.19(3)$ & $2.00(5)$ \\
\hline$E M_{1}\left[10^{49} \mathrm{~cm}^{-3}\right]$ & $1.01(11)$ & $1.08(10)$ \\
\hline$E M_{2}\left[10^{49} \mathrm{~cm}^{-3}\right]$ & $1.87(17)$ & $1.11(8)$ \\
\hline$E M_{\mathrm{tot}}\left[10^{49} \mathrm{~cm}^{-3}\right]$ & $2.88(19)$ & $2.09(13)$ \\
\hline$L_{x}\left[10^{27} \mathrm{ergs}\right](0.07-2.5 \mathrm{keV})$ & $2.4(3)$ & $2.3(3)$ \\
\hline$L_{x}\left[10^{27}\right.$ ergs $](0.1-2.4 \mathrm{keV})$ & 1.9 & 1.6 \\
\hline$L_{x}\left[10^{27}\right.$ ergs $](0.15-4 \mathrm{keV})$ & 1.6 & 0.87 \\
\hline $\mathrm{C} / \mathrm{Fe} 11.26 \mathrm{eV}$ & $0.44(9)$ & $0.58(9)$ \\
\hline $\mathrm{N} / \mathrm{Fe} 14.53 \mathrm{eV}$ & $0.30(12)$ & $0.46(11)$ \\
\hline $\mathrm{O} / \mathrm{Fe} 13.62 \mathrm{eV}^{c}$ & $0.23(4)$ & $0.30(5)$ \\
\hline $\mathrm{Ne} / \mathrm{Fe} 21.56 \mathrm{eV}^{c}$ & $0.38(13)$ & $0.37(13)$ \\
\hline $\mathrm{Mg} / \mathrm{Fe} 7.65 \mathrm{e} \mathrm{V}^{c}$ & $1.12(14)$ & $1.01(21)$ \\
\hline $\mathrm{Si} / \mathrm{Fe} 8.15 \mathrm{eV}^{c}$ & $0.86(12)$ & $1.18(16)$ \\
\hline $\mathrm{Fe} / \mathrm{Fe} 7.87 \mathrm{eV}$ & 1 & 1 \\
\hline $\mathrm{Ni} / \mathrm{Fe} 7.64 \mathrm{eV}$ & $1.67(26)$ & $1.55(20)$ \\
\hline $\mathrm{Fe} / \mathrm{H}^{c}$ & $1.43(9)$ & $1.36(16)$ \\
\hline$\chi_{\text {red }}^{2} /$ dof & $6130 / 4484$ & $5887 / 4453$ \\
\hline
\end{tabular}

${ }^{a}$ In logarithmic units, with $\log _{10} H=12.00 ; \mathrm{C}=8.56 ; \mathrm{N}=8.05$; $\mathrm{O}=8.93 ; \mathrm{Ne}=8.09 ; \mathrm{Mg}=7.58$;

$\mathrm{Si}=7.55 ; \mathrm{S}=7.21 ; \mathrm{Ar}=6.56 ; \mathrm{Ca}=6.36 ; \mathrm{Fe}=7.51$ (see text); $\mathrm{Ni}=6.25$.

${ }^{b}$ From Mewe et al. (1995b).

${ }^{c}$ Corrected abundance ratios from ASCA obs. (Mewe et al. 1998): $\mathrm{O} / \mathrm{Fe}=0.3(1), \mathrm{Ne} / \mathrm{Fe}=0.7(2), \mathrm{Mg} / \mathrm{Fe}=3.0(7), \mathrm{Si} / \mathrm{Fe}=4(3)$, $\mathrm{Fe} / \mathrm{H}=1.1(4)$.

code in some wavelength ranges and to the high spectral resolution of the instrument. Small wavelength deviations (about 1-2 bins, i.e., 0.02-0.04 $\AA$ ) between lines in the spectrum and in the model are often present. In Table 3 results for temperatures $T$ (in $\mathrm{MK}$ ), emission measures $E M$ and abundances of the $\mathrm{K} 1 \mathrm{~V}$ and $\mathrm{G} 2 \mathrm{~V}$ star are given, together with the ionization potential of the neutral ion of each element (the First Ionization Potential). The emission measure is defined as $E M=n_{\mathrm{e}} n_{\mathrm{H}} V$, where $V$ is the volume contributing to the emission and for solar abundances the hydrogen density $n_{\mathrm{H}} \simeq 0.85 n_{\mathrm{e}}$. The abundances are compared to the iron abundance and relative to solar photospheric values (Anders \& Grevesse 1989) except for Fe. For that element we use $\log A_{\mathrm{Fe}}=7.50$ (see Grevesse \& Sauval $1998,1999)$ instead of 7.67. Though a determination of the Fe abundance in the photosphere in $\alpha$ Cen may yield a value of 

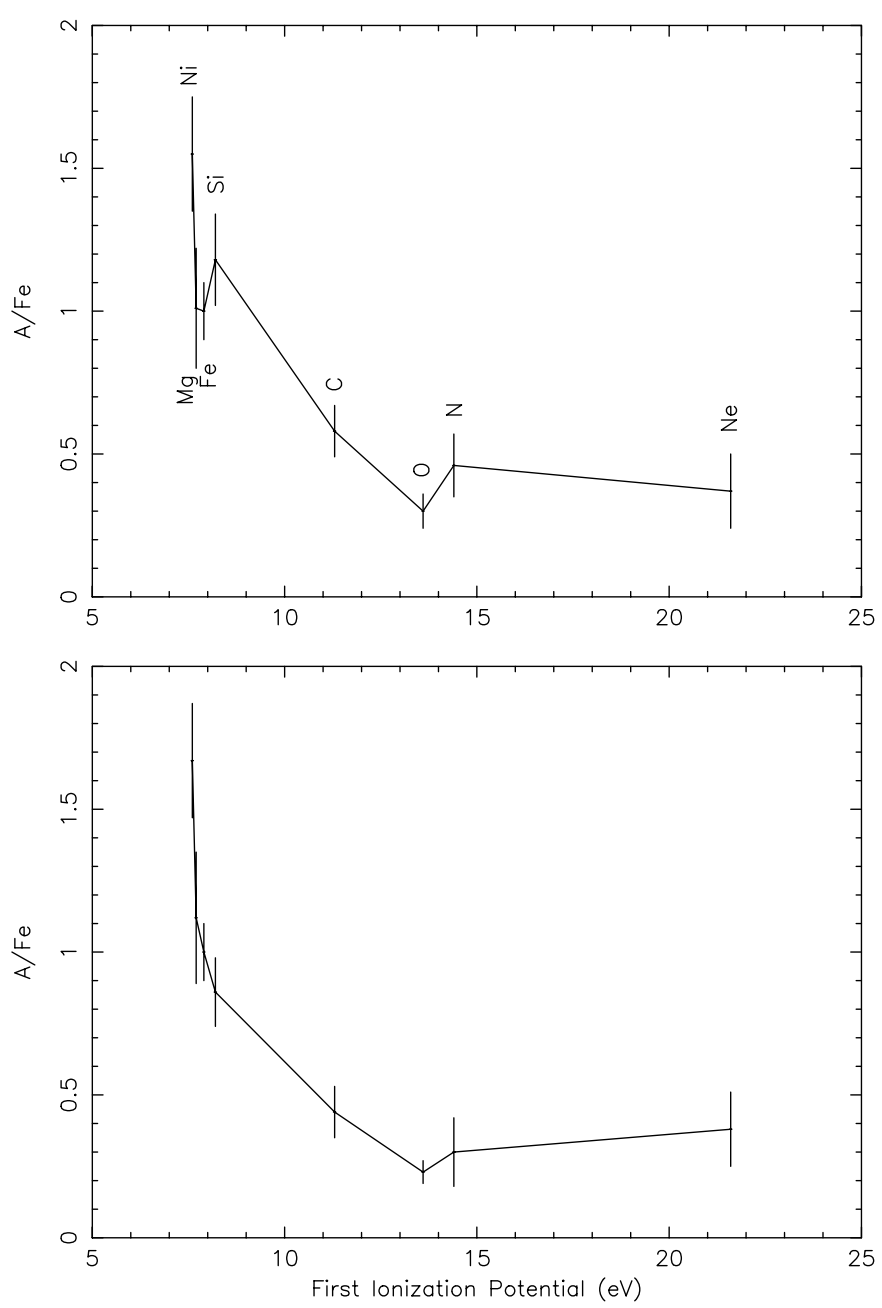

Fig. 4. Abundances relative to iron for the G2V star (top) and K1V star (bottom) of $\alpha$ Centauri.

about 7.70 (Smith et al. 1986) we prefer in view of the uncertainties (e.g., Drake et al. 1997) to use the recent solar value of Grevesse \& Sauval. For the other elements the abundances of Anders \& Grevesse are within the errors consistent with those from Grevesse \& Sauval (but see the discussion on the oxygen abundance in the next paragraph). Statistic $1 \sigma$ uncertainties are given in parentheses. The two temperatures and emission measures of both spectra are between 1 and 2.5 MK. This is comparable to the average value of 2.1 MK obtained by Golub et al. (1982) with the Einstein IPC detector. But the K1V star has a higher value for the $E M$ at higher temperature. Due to the strong correlation between the absolute abundance values and the emission measure, abundance values are given relative to the iron abundance value.

From Table 3 and Fig. 4 we notice the relatively high abundances for the elements with a low First Ionization Potential, indicating a FIP effect. This FIP effect was first observed in the solar corona (e.g., Feldman et al. 1992). For hot active stars also an inverse FIP effect is observed (Güdel et al. 2003). This confirms the work by Drake et al. (1997), based on EUVE observations of the $\alpha$ Cen ensemble. The low value of oxygen also

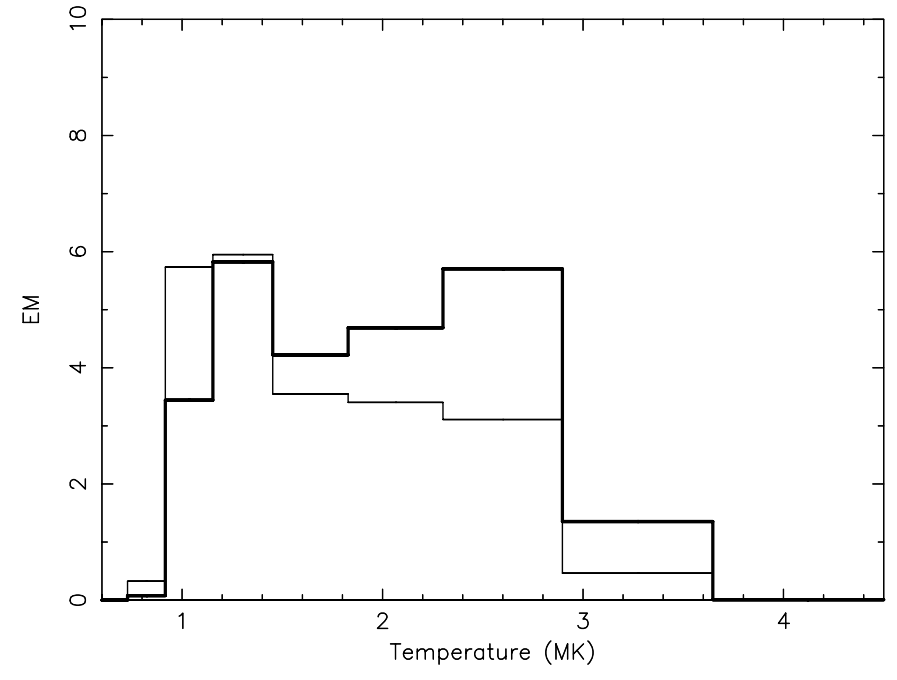

Fig. 5. DEM modeling of the G2V star (thin line) and the K1V star (thick line) of $\alpha$ Centauri. $E M=n_{\mathrm{e}} n_{\mathrm{H}} V$ in units $10^{48} \mathrm{~cm}^{-3}$ and per logarithmic temperature bin.

appears in Fig. 7 of Drake et al. (1997) and might be due to deficiences of the used solar oxygen abundance. Allende Prieto \& Lambert (2001) have found a lower solar photospheric oxygen abundance value of $\log A_{\mathrm{O}}=8.69$ (instead of $\log A_{\mathrm{O}}=8.93$ ) bringing the oxygen value in line with nitrogen and carbon. The abundance ratios from ASCA observations (Mewe et al. 1998), reduced by a factor 1.48 because they use $\log A_{\mathrm{Fe}}=7.67$ (instead of 7.51), are within the statistical errors comparable to our values (cf. Table 3). Comparing the abundance values in Table 3 (especially those of Ne (high FIP) and Mg and Ni (low FIP)) some indication is found for a stronger FIP effect in the $\mathrm{K} 1 \mathrm{~V}$ star than in the G2V star. However, this indication is not very significant in view of the uncertainties.

To show the connectivity of the different temperature components obtained in the multi-temperature fitting, we applied a differential emission measure (DEM) model of the coronae of the $\mathrm{K} 1 \mathrm{~V}$ and the $\mathrm{G} 2 \mathrm{~V}$ stars of $\alpha \mathrm{Cen}$, using the various inversion techniques offered by SPEX (see Kaastra et al. 1996b). We applied the abundances obtained in the multi-temperature fit of Table 3. Figure 5 shows the $E M$ based on the polynomial method of order 10 . The temperatures range from 0.9 up to $3.5 \mathrm{MK}$ for the $\mathrm{G} 2 \mathrm{~V}$ and the $\mathrm{K} 1 \mathrm{~V}$ star. From this figure it is clear that the G2V star (thin line) has a lower emission measure volume at the higher coronal temperature than the K1V star (thick line).

In Table A. 2 the measured line fluxes of a number of selected lines have been compared with the model fluxes obtained from the DEM-modeling and abundances given in Table 3. Taking into account the uncertainties in the atomic data $(20-$ $50 \%$ ) the flux values are in good agreement with each other. From this table we notice that the modeled fluxes for the H-like ions ( $\mathrm{C}$ VI, $\mathrm{N}$ VII and $\mathrm{O}$ VIII) are higher than the measured line fluxes, while those for the He-like ions ( $\mathrm{C} \mathrm{v}, \mathrm{N}$ vI, and $\mathrm{O}$ vII) are lower. This might indicate a systematic error in atomic data or the wrong assumption that the elemental abundances has to be coupled for all temperature bins. The latter might imply that 
the He-like lines are formed in inactive regions of the corona and the $\mathrm{H}$-like lines in active regions, resulting in higher abundances for He-like ions compared to H-like ions.

\section{Conclusions}

The coronal spectra of the two components of $\alpha$ Centauri (K1V and G2V) are analyzed separately for the first time. Both LETGS spectra are full with emission lines, but no significant continuum emission can be measured. We use the strongest emission lines in order to derive temperatures and to constrain densities for each component. In addition we construct selfconsistent emission measure distributions for the stars in order to derive elemental abundances. This is done using global fitting of multi- $T$ models with a combination of SPEX and MEKAL databases.

We use the He-like $f / i$ ratio of oxygen, nitrogen, and carbon in order to constrain the plasma densities. For the G2V star we find low density limits with all the ions. They are all consistent the low density limit found for carbon, indicating the density below $2 \times 10^{9} \mathrm{~cm}^{-3}$. For the K1V star the intercombination line of oxygen is not detected and the low density limit is measured with nitrogen, which is, again, consistent with the measurement of carbon, indicating a density range of $0.5-3 \times 10^{9} \mathrm{~cm}^{-3}$. We point out the the carbon $f / i$ ratio measures at the lowest densities, but has the highest low density value of $f / i$ of all the ions. Therefore the intercombination line is very weak and very long exposure times are necessary in order to better constrain the densities measured with the carbon $f / i$ ratio.

Plasma temperatures are measured using line flux ratios of $\mathrm{H}$-like and He-like lines of oxygen, nitrogen, and carbon. We find temperatures between 1 and $2 \mathrm{MK}$ with a slight but not significant tendency that the K1V star is hotter than the G2V star (Table 2). However, this tendency is much clearer when comparing line flux ratios of the same lines for the two stars. We find that the lines formed at lower temperatures (Fe Ix, Fex, $\mathrm{C}$ v, N vi, Si vIII, Si Ix, and Si x) are stronger in the G2V star, while the "hotter" lines of Fe xI, N vII, O VII, O vIII, Si XI, and Si XII are stronger in the K1V star. From these measurements we conclude that in the $\mathrm{G} 2 \mathrm{~V}$ star the emission measure distribution peaks at lower temperatures than in the K1V star. The emission measure distributions derived from the DEM modeling are consistent with this trend and we conclude that they can be used for further analysis expecting results consistent with our analysis from individual lines. We calculated the abundances relative to iron for the two stars and find a FIP effect for both stars. Some indication for a stronger FIP effect in the corona of the K1V star than in the G2V star may be present. However, the errors for these measurements are dominated by systematic effects such as misplacements in wavelength or a lack of lines in the databases, so that the given statistical errors are not fully representative.

Acknowledgements. The National Institute for Space Research (SRON) is financially supported by NWO. We are grateful to the Chandra team, that is responsible for the on board calibration of the LETGS. We like to thank the referee for useful comments.

\section{Appendix A:}

Table A.1. Wavelengths and fluxes ( $1 \sigma$ error in parentheses) from LETGS for the $\alpha$ Cen K1V and G2V star.

\begin{tabular}{|c|c|c|c|c|}
\hline \multicolumn{2}{|c|}{$\alpha \operatorname{Cen}(\mathrm{K} 1 \mathrm{~V})$} & \multicolumn{2}{|c|}{$\alpha \operatorname{Cen}(\mathrm{G} 2 \mathrm{~V})$} & Line ID $^{a}$ \\
\hline$\lambda(\AA)$ & flux $^{b}$ & $\lambda(\AA)$ & flux $^{b}$ & $\lambda(\AA) \quad$ Ion \\
\hline $13.439(18)$ & $0.09(4)$ & $13.462(11)$ & $0.06(3)$ & $13.447 \mathrm{Ne}$ IX \\
\hline- & - & $13.658(11)$ & 0.02 (3) & $13.700 \mathrm{Ne} I X$ \\
\hline $15.011(17)$ & 0.14 & 15.027 (12) & $0.15(4)$ & 15.013 Fe XVII \\
\hline $15.220(15)$ & 0.12 & 15.243 (13) & $0.10(4)$ & 15.260 Fe XVII \\
\hline $15.991(27)$ & 0.08 & $15.991(12)$ & $0.05(3)$ & $16.007 \mathrm{O}$ VIII \\
\hline $16.780(56)$ & 0.13 & $16.781(11)$ & 0.08 (4) & 16.775 Fe XVII \\
\hline $17.040(14)$ & 0.12 & $17.075(9)$ & 0.11 (4) & 17.051 Fe XVII \\
\hline 17.093 & 0.19 & $17.115(9)$ & $0.06(3)$ & 17.100 Fe XVII \\
\hline $18.630(10$ & 0.10 & $18.638(12)$ & $0.10(4)$ & $18.627 \mathrm{O}$ VII \\
\hline $18.974(5)$ & $0.53(7)$ & $18.968(5)$ & $0.27(5)$ & $18.969 \mathrm{O}$ VIII \\
\hline $20.875(11)$ & 0.14 & - & - & 20.910 N VII \\
\hline $21.606(4)$ & $1.09(11)$ & 21.601 & $0.84(10)$ & $21.602 \mathrm{O}$ VII \\
\hline $21.808(9)$ & $0.15(5)$ & $21.812(9)$ & $0.18(5)$ & $21.804 \mathrm{O}$ VII \\
\hline 22.098 (4) & $1.02(11)$ & 22.095 & $0.66(9)$ & $22.101 \mathrm{O}$ VII \\
\hline 24.787 (9) & $0.20(6)$ & $24.795(11)$ & $0.10(4)$ & $24.781 \mathrm{~N}$ VII \\
\hline $28.444(18)$ & 0.11 & $28.482(10)$ & $0.10(4)$ & 28.466 C VI \\
\hline $28.799(51)$ & 0.23 & $28.782(9)$ & $0.24(6)$ & 28.787 N VI \\
\hline $29.101(12)$ & 0.07 & 29.085 (11) & 0.07 (3) & 29.084 N VI \\
\hline $29.533(9)$ & $0.20(6)$ & 29.539 (10) & $0.28(7)$ & $29.534 \mathrm{~N} \mathrm{VI}$ \\
\hline $33.520(12)$ & 0.21 & $33.520(10)$ & $0.13(5)$ & 33.515 Si XI \\
\hline 33.739 (5) & $1.22(11)$ & $33.742(4)$ & $1.21(11)$ & $33.736 \mathrm{C} \mathrm{VI}$ \\
\hline- & - & $35.000(9)$ & $0.18(6)$ & $34.973 \mathrm{C} \mathrm{V}$ \\
\hline $35.600(9)$ & $0.25(7)$ & $35.587(8)$ & $0.21(6)$ & $35.576 \mathrm{Ca} \mathrm{XI}$ \\
\hline $35.697(7)$ & $0.38(8)$ & $35.660(12)$ & $0.18(6)$ & 35.665 S XIII \\
\hline 40.264 (11) & 0.45 & $40.244(8)$ & $0.77(12)$ & $40.268 \mathrm{C} \mathrm{V}$ \\
\hline- & - & 40.414 (13) & 0.08 (4) & - \\
\hline- & - & $40.634(12)$ & $0.15(6)$ & - \\
\hline 40.704(15) & $0.13(5)$ & 40.749 (15) & $0.25(8)$ & $40.731 \mathrm{C} \mathrm{V}$ \\
\hline $40.872(11)$ & 0.37 & & - & - \\
\hline $41.456(48)$ & 0.49 & 41.461 & $0.68(11)$ & $41.472 \mathrm{C} \mathrm{V}$ \\
\hline $43.668(11)$ & 0.18 & - & - & - \\
\hline $43.760(5)$ & $0.51(7)$ & 43.748 (7) & $0.43(6)$ & $43.763 \mathrm{Si}$ XI \\
\hline $43.915(8)$ & $0.14(5)$ & $43.900(10)$ & $0.13(4)$ & - \\
\hline $44.046(8)$ & $0.31(6)$ & $44.042(10)$ & $0.19(5)$ & $\begin{array}{l}44.050 \mathrm{Mg} \mathrm{X} \\
\text { 44.020 Si XII }\end{array}$ \\
\hline $44.164(6)$ & $0.52(7)$ & $44.155(6)$ & $0.41(5)$ & 44.165 Si XII \\
\hline 44.244 (12) & 0.20 & $44.211(6)$ & $0.26(5)$ & 44.215 Si IX \\
\hline $45.511(8)$ & $0.19(5)$ & $45.518(9)$ & $0.16(4)$ & 45.519 Si XII \\
\hline $45.691(9)$ & $0.27(5)$ & $45.692(8)$ & $0.21(5)$ & 45.692 Si XII \\
\hline $46.278(12)$ & $0.19(5)$ & $46.287(9)$ & $0.21(5)$ & 46.300 Si XI \\
\hline $46.401(8)$ & $0.22(5)$ & $46.393(8)$ & $0.40(7)$ & 46.401 Si XI \\
\hline 47.238 (10) & 0.15 & - & - & $47.231 \mathrm{Mg} \mathrm{X}$ \\
\hline $47.640(15)$ & 0.18 & 47.645 (11) & $0.16(4)$ & $47.655 \mathrm{~S} \mathrm{X}$ \\
\hline $47.802(12)$ & 0.20 & 47.837 (10) & $0.13(4)$ & $47.791 \mathrm{~S} \mathrm{X}$ \\
\hline 47.931 (12) & 0.14 & - & - & $47.905 \mathrm{~S} X$ \\
\hline $49.201(5)$ & $0.94(8)$ & $49.204(6)$ & $0.80(8)$ & 49.222 Si XI \\
\hline 49.691 (11) & 0.13 & - & - & 49.701 Si X \\
\hline $50.324(7)$ & $0.37(8)$ & $50.321(9)$ & $0.31(6)$ & 50.333 Si X \\
\hline $50.513(6)$ & $0.89(12)$ & 50.515 & 0.99 (10) & $50.524 \mathrm{Si} \mathrm{X}$ \\
\hline $50.682(5)$ & $0.78(10)$ & $50.679(6)$ & $0.91(11)$ & $50.691 \mathrm{Si} \mathrm{X}$ \\
\hline $52.314(6)$ & $0.68(11)$ & 52.331 & $0.34(7)$ & 52.296 Si XI \\
\hline $52.876(10)$ & 0.27 & $52.915(10)$ & $0.34(7)$ & $52.911 \mathrm{Fe} X V$ \\
\hline $54.723(9)$ & $0.35(8)$ & - & - & 54.728 Fe XVI \\
\hline- & - & 55.091 (19) & $0.32(7)$ & $\begin{array}{l}55.096 \mathrm{Si} \mathrm{X} \\
55.060 \mathrm{Mg} \text { IX }\end{array}$ \\
\hline
\end{tabular}

${ }^{a}$ Line identification from KELLY (1987).

${ }^{b}$ Observed flux at Earth in $10^{-4}$ photons $\mathrm{cm}^{-2} \mathrm{~s}^{-1}$. 
Table A.1. continued.

\begin{tabular}{|c|c|c|c|c|}
\hline \multicolumn{2}{|c|}{$\alpha$ Cen (K1V) } & \multicolumn{2}{|c|}{$\alpha$ Cen (G2V) } & 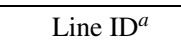 \\
\hline$\lambda(\AA)$ & flux $^{b}$ & $\lambda(\AA)$ & flux $^{b}$ & $\lambda(\AA) \quad$ Ion \\
\hline $55.331(7)$ & $0.55(10)$ & $55.301(8)$ & $0.80(11)$ & $55.305 \mathrm{Si}$ IX \\
\hline $55.393(7)$ & $0.90(12)$ & $55.380(8)$ & $1.22(14)$ & $55.356 \mathrm{Si}$ IX \\
\hline - & - & - & - & $55.401 \mathrm{Si}$ IX \\
\hline $56.920(12)$ & 0.28 & $56.949(9)$ & $0.46(8)$ & - \\
\hline $57.202(8)$ & $0.43(9)$ & $57.182(9)$ & $0.38(7)$ & - \\
\hline $57.365(10)$ & $0.32(7)$ & $57.359(9)$ & $0.42(7)$ & - \\
\hline $57.869(7)$ & $0.65(9)$ & $57.873(6)$ & $0.85(9)$ & $57.876 \mathrm{Mg} \mathrm{X}$ \\
\hline $58.891(11)$ & 0.17 & $58.876(11)$ & $0.24(6)$ & - \\
\hline $58.956(12)$ & $0.37(7)$ & $58.928(11)$ & $0.32(7)$ & $58.963 \mathrm{Fe}$ XIV \\
\hline $59.121(10)$ & $0.23(6)$ & $59.160(11)$ & $0.21(5)$ & $59.153 \mathrm{Mg}$ VIII \\
\hline $59.391(7)$ & $0.46(9)$ & $59.394(9)$ & $0.29(6)$ & - \\
\hline $59.596(10)$ & $0.16(5)$ & $59.596(12)$ & $0.15(4)$ & - \\
\hline $60.800(12)$ & $0.33(8)$ & - & - & - \\
\hline $60.981(11)$ & $0.39(10)$ & ) $61.011(11)$ & $0.88(11)$ & 61.019 Si VIII \\
\hline $61.069(7)$ & $0.85(12)$ & 61.067(11) & $1.04(12)$ & 61.070 Si VIII \\
\hline $61.638(10)$ & 0.59 & ) $61.686(11)$ & $0.57(11)$ & $61.649 \mathrm{Si}$ IX \\
\hline $61.925(28)$ & 0.54 & ) $61.918(10)$ & $0.93(13)$ & 61.914 Si VIII \\
\hline 63.163 (11) & 0.19 & 63.129 & 0.24 & $63.152 \mathrm{Mg} X$ \\
\hline $63.294(6)$ & $0.97(11)$ & $63.280(8)$ & $0.85(12)$ & $63.295 \mathrm{Mg} \mathrm{X}$ \\
\hline- & - & $63.894(12)$ & $0.43(10)$ & 63.903 Si VIII \\
\hline $65.667(12)$ & $0.39(8)$ & $65.675(10)$ & $0.38(9)$ & $65.672 \mathrm{Mg} \mathrm{X}$ \\
\hline $65.812(13)$ & 0.15 & $65.826(9)$ & $0.53(9)$ & $65.822 \mathrm{Ne}$ VIII \\
\hline $65.867(12)$ & $0.59(9)$ & & & $65.847 \mathrm{Mg} \mathrm{X}$ \\
\hline $67.166(11)$ & $0.40(6)$ & $67.156(12)$ & $0.29(6)$ & $67.135 \mathrm{Mg}$ IX \\
\hline $67.245(10)$ & $0.46(7)$ & $67.246(10)$ & $0.57(9)$ & $67.239 \mathrm{Mg} I X$ \\
\hline $67.347(10)$ & 0.50 & $67.365(9)$ & $0.34(6)$ & $67.382 \mathrm{Ne}$ VIII \\
\hline $69.672(5)$ & $1.45(12)$ & $69.661(7)$ & $1.46(11)$ & $69.632 \mathrm{Si}$ VIII \\
\hline 70.039 (9) & $0.55(8)$ & $70.036(9)$ & $0.44(7)$ & $70.054 \mathrm{Fe} X \mathrm{~V}$ \\
\hline 71.924 (7) & $0.68(10)$ & 71.918 (29) & $0.51(7)$ & $71.901 \mathrm{Mg}$ IX \\
\hline $72.041(10)$ & $0.30(6)$ & $72.029(25)$ & $0.39(7)$ & $72.027 \mathrm{Mg}$ IX \\
\hline $72.318(28)$ & 0.55 & $72.322(20)$ & $0.71(10)$ & $72.312 \mathrm{Mg}$ IX \\
\hline $73.481(7)$ & $0.71(11)$ & ) 73.479 (12) & $0.37(6)$ & $73.470 \mathrm{Ne}$ VIII \\
\hline- & - & $74.379(11)$ & $0.35(6)$ & - \\
\hline 74.865 (7) & $0.55(10)$ & 74.878 (7) & $0.76(10)$ & $74.858 \mathrm{Mg}$ VIII \\
\hline $75.060(6)$ & $0.69(11)$ & $75.050(6)$ & $1.13(12)$ & $75.034 \mathrm{Mg}$ VIII \\
\hline 75.905 (14) & $0.36(8)$ & - & - & - \\
\hline $76.033(6)$ & $1.01(11)$ & $76.042(10)$ & $0.40(7)$ & 76.022 Fe XIV \\
\hline $76.143(10)$ & 0.68 & 76.158 (11) & $0.30(6)$ & 76.152 Fe XIV \\
\hline & & & & 76.117 Fe XIII \\
\hline 76.688 (12) & $0.32(8)$ & - & - & - \\
\hline 76.877 (7) & $0.69(11)$ & $76.877(9)$ & $0.44(7)$ & - \\
\hline $76.991(10)$ & 0.35 & - & - & - \\
\hline $77.373(11)$ & $0.29(7)$ & - & - & $77.393 \mathrm{Ni}$ XI \\
\hline 77.518 (10) & $0.30(7)$ & - & - & - \\
\hline $77.756(7)$ & $0.66(10)$ & $77.751(9)$ & $0.66(9)$ & $77.737 \mathrm{Mg}$ IX \\
\hline- & - & $77.872(14)$ & 0.44 & $77.865 \mathrm{Fe} \mathrm{X}$ \\
\hline $78.738(9)$ & $0.40(8)$ & $78.762(10)$ & $0.38(7)$ & 78.744 Ni XI \\
\hline $79.482(9)$ & $0.50(9)$ & $79.479(10)$ & $0.36(7)$ & 79.488 Fe XII \\
\hline $82.430(8)$ & $0.57(9)$ & $82.423(12)$ & $0.32(7)$ & $82.430 \mathrm{Fe}$ IX \\
\hline $82.660(7)$ & $0.83(11)$ & $82.665(30)$ & $0.62(9)$ & $82.598 \mathrm{Mg}$ VIII \\
\hline- & - & $82.804(30)$ & $0.37(7)$ & $82.822 \mathrm{Mg}$ VIII \\
\hline $83.204(9)$ & $0.42(8)$ & - & - & - \\
\hline
\end{tabular}

Table A.1. continued.

\begin{tabular}{|c|c|c|c|c|c|}
\hline \multicolumn{2}{|c|}{$\alpha$ Cen $(\mathrm{K} 1 \mathrm{~V})$} & \multicolumn{2}{|c|}{$\alpha$ Cen (G2V) } & \multicolumn{2}{|c|}{ Line $\mathrm{ID}^{a}$} \\
\hline$\lambda(\AA)$ & flux $^{b}$ & $\lambda(\AA)$ & flux $^{b}$ & $\lambda(\AA)$ & Ion \\
\hline $83.386(10)$ & $0.37(7)$ & $83.342(30)$ & $0.40(7)$ & 83.358 & Si VI \\
\hline- & - & $83.443(30)$ & $0.41(7)$ & 83.457 & Fe IX \\
\hline - & - & $83.571(30)$ & $0.52(9)$ & 83.587 & Mg VII \\
\hline - & - & $83.959(30)$ & $0.44(8)$ & 83.959 & Mg VII \\
\hline - & - & $84.054(30)$ & $0.60(9)$ & 84.025 & Mg VII \\
\hline 85.444 (8) & $0.66(11)$ & $85.448(12)$ & $0.26(7)$ & 85.461 & Fe XIII \\
\hline $86.758(8)$ & $0.55(9)$ & 86.767 (18) & $0.30(7)$ & 86.772 & Fe XI \\
\hline - & - & 88.094 (10) & $0.19(5)$ & 88.092 & Ne VIII \\
\hline 88.936 (7) & $0.60(9)$ & $88.930(8)$ & 0.64 (11) & 88.952 & Mg VI \\
\hline 89.170 (10) & $0.59(9)$ & $89.144(12)$ & $0.25(8)$ & 89.185 & Fe XI \\
\hline 90.189 (10) & $0.43(9)$ & $90.201(10)$ & $0.42(10)$ & & \\
\hline 90.707 (10) & $0.35(8)$ & - & - & 90.708 & $\mathrm{Mg}$ VII \\
\hline 90.995 (9) & $0.44(9)$ & - & - & 91.009 & Fe XIV \\
\hline $91.563(10)$ & $0.30(7)$ & $91.529(12)$ & $0.36(9)$ & 91.527 & Ni X \\
\hline 91.789 (11) & $0.60(10)$ & $91.817(12)$ & $0.54(11)$ & 91.790 & Ni X \\
\hline 92.186 (9) & $0.48(9)$ & $92.148(12)$ & $0.30(10)$ & 92.123 & Mg VIII \\
\hline $94.000(7)$ & $1.26(13)$ & $94.012(7)$ & $1.10(13)$ & 94.012 & $\mathrm{Fe} X$ \\
\hline - & - & $95.359(12)$ & $0.37(10)$ & 95.338 & Fe X \\
\hline - & - & $95.440(10)$ & $0.52(11)$ & 95.421 & Mg VI \\
\hline 95.856 (11) & $0.35(8)$ & - & - & - & \\
\hline $95.992(8)$ & $0.58(10)$ & $95.997(8)$ & $0.89(11)$ & 96.022 & Si VI \\
\hline $96.101(8)$ & $0.69(11)$ & 96.100 & $0.21(6)$ & 96.122 & $\mathrm{Fe} X$ \\
\hline $98.093(9)$ & $0.36(10)$ & 98.120 & $0.79(15)$ & 98.115 & Ne VIII \\
\hline $98.242(48)$ & $0.49(11)$ & 98.262 & $0.40(11)$ & 98.260 & $\mathrm{Ne}$ VIII \\
\hline - & - & $98.495(12)$ & $0.83(18)$ & - & \\
\hline - & - & $100.005(10)$ & $0.40(10)$ & - & \\
\hline $100.567(6)$ & $0.95(13)$ & 100.561 & $1.06(15)$ & 100.597 & 7 Mg VIII \\
\hline- & - & $102.765(13)$ & $0.62(14)$ & - & \\
\hline $103.039(22)$ & $0.53(27)$ & 103.063 & $0.65(12)$ & 103.08 & 5 Ne VIII \\
\hline $103.262(43)$ & $0.49(28)$ & 103.280 & $0.41(12)$ & & \\
\hline 103.567 (19) & $0.68(29)$ & 103.537 & $0.78(12)$ & 103.566 & $6 \mathrm{Fe} \mathrm{IX}$ \\
\hline $103.926(40)$ & $0.53(29)$ & $103.904(12)$ & $0.49(12)$ & & \\
\hline- & - & $104.856(11)$ & $0.72(14)$ & 104.813 & $3 \mathrm{O} \mathrm{VI}$ \\
\hline $105.183(11)$ & $0.56(10)$ & 105.197 & $1.06(16)$ & 105.208 & 8 Fe IX \\
\hline $108.420(46)$ & $0.88(12)$ & & - & - & \\
\hline- & - & $116.675(11)$ & $0.38(9)$ & 116.693 & $3 \mathrm{Ne} \mathrm{VII}$ \\
\hline - & - & $122.482(10)$ & $0.71(13)$ & 122.49 & $\mathrm{Ne} V I$ \\
\hline $129.818(13)$ & $1.42(22)$ & & - & 129.871 & $1 \mathrm{O} \mathrm{VI}^{c}$ \\
\hline $142.378(11)$ & $1.42(18)$ & & - & - & \\
\hline 146.095 (12) & 1.38 & & - & - & \\
\hline 148.339 (6) & $6.05(44)$ & $148.332(6)$ & $6.54(46)$ & 148.402 & $2 \mathrm{Ni} \mathrm{XI}$ \\
\hline - & - & $150.046(11)$ & $2.19(23)$ & 150.089 & $9 \mathrm{O} \mathrm{VI}$ \\
\hline - & - & 150.099 & 1.38 & 150.124 & $4 \mathrm{O}$ VI \\
\hline - & - & $150.332(14)$ & $1.26(18)$ & - & \\
\hline $152.110(39)$ & 5.40 & 152.120 & $3.66(34)$ & 152.153 & 3 Ni XII \\
\hline $154.131(10)$ & $2.36(28)$ & 154.158 & $1.03(15)$ & 154.175 & $5 \mathrm{Ni}$ XII \\
\hline $157.596(12)$ & $1.87(25)$ & & - & - & \\
\hline $157.730(9)$ & $3.24(35)$ & 157.709 & $1.96(23)$ & 157.730 & 0 Ni XIII \\
\hline - & - & $157.972(30)$ & $1.31(30)$ & & \\
\hline 171.089 (4) & $51.8(25)$ & 171.093 & 71.7 (29) & 171.075 & $5 \mathrm{Fe}$ IX \\
\hline $174.535(6)$ & $50.3(43)$ & 174.537 & $52.2(43)$ & 174.53 & $4 \mathrm{Fe} \mathrm{X}$ \\
\hline
\end{tabular}

${ }^{c}$ Line identification unlikely due to the absence of this line in the spectrum of the G2V star. 
Table A.2. Comparison of model and observed fluxes from LETGS for the $\alpha$ Cen K1V and G2V star.

\begin{tabular}{|c|c|c|c|c|c|}
\hline \multirow{2}{*}{\multicolumn{2}{|c|}{$\frac{\alpha \text { Cen }(\mathrm{K} 1 \mathrm{~V})}{\text { flux }^{b} \text { flux }^{c}}$}} & \multicolumn{2}{|c|}{$\alpha$ Cen $(\mathrm{G} 2 \mathrm{~V})$} & \multicolumn{2}{|c|}{ Line $\mathrm{ID}^{a}$} \\
\hline & & flux $^{b}$ & & $\lambda(\AA)$ & Ion \\
\hline 0.41 & $0.45(10)$ & 0.56 & 0.77 (12) & 40.268 & $\mathrm{C} \mathrm{V}$ \\
\hline 0.03 & $0.13(5)$ & 0.04 & $0.25(8)$ & 40.731 & $\mathrm{C} \mathrm{V}$ \\
\hline 0.37 & 0.49 (11) & 0.51 & 0.68 (11) & 41.472 & $\mathrm{C} \mathrm{V}$ \\
\hline 0.11 & & 0.14 & & 41.480 & Ar IX \\
\hline 0.12 & $0.11(4)$ & 0.10 & $0.10(4)$ & 28.466 & C VI \\
\hline 1.25 & $1.22(11)$ & 1.23 & $1.21(11)$ & 33.736 & C VI \\
\hline 0.16 & $0.23(6)$ & 0.17 & $0.24(6)$ & 28.787 & N VI \\
\hline 0.02 & $0.07(4)$ & 0.03 & $0.07(3)$ & 29.084 & N VI \\
\hline 0.12 & $0.20(6)$ & 0.14 & $0.28(7)$ & 29.534 & N VI \\
\hline 0.20 & $0.20(6)$ & 0.16 & $0.10(4)$ & 24.781 & N VII \\
\hline 0.10 & $0.10(4)$ & 0.08 & $0.10(4)$ & 18.627 & O VII \\
\hline 0.92 & 1.09 (11) & 0.78 & $0.84(10)$ & 21.602 & O VII \\
\hline 0.16 & $0.15(5)$ & 0.14 & $0.18(5)$ & 21.804 & O VII \\
\hline 0.63 & $1.02(11)$ & 0.56 & $0.66(9)$ & 22.101 & O VII \\
\hline 0.05 & 0.08 & 0.03 & $0.05(3)$ & 16.007 & O VIII \\
\hline 0.59 & $0.53(7)$ & 0.36 & $0.27(5)$ & 18.969 & O VIII \\
\hline 0.07 & $0.09(4)$ & 0.03 & $0.06(3)$ & 13.447 & $\mathrm{Ne} I X$ \\
\hline 0.30 & $0.55(10)$ & 0.47 & $0.76(10)$ & 74.858 & Mg VIII \\
\hline 0.27 & & 0.25 & & 74.843 & Fe XIII \\
\hline 0.35 & $0.69(11)$ & 0.55 & $1.13(12)$ & 75.034 & Mg VIII \\
\hline 0.18 & $0.40(6)$ & 0.21 & $0.29(6)$ & 67.135 & Mg IX \\
\hline 0.55 & $0.30(6)$ & 0.72 & $0.39(7)$ & 72.027 & Mg IX \\
\hline 0.40 & $0.55(9)$ & 0.51 & 0.71 & 72.311 & Mg IX \\
\hline 0.19 & & 0.21 & & 72.310 & Fe IX \\
\hline \multirow[t]{2}{*}{0.90} & $1.24(16)$ & 1.45 & $1.92(25)$ & 61.019 & Si VIII \\
\hline & & & & 61.070 & Si VIII \\
\hline 0.22 & $0.20(5)$ & 0.19 & $0.26(5)$ & 44.215 & Si IX \\
\hline 0.27 & $0.55(10)$ & 0.35 & $0.80(11)$ & 55.305 & Si IX \\
\hline 1.64 & $0.90(12)$ & 2.15 & $1.22(14)$ & 55.356 & Si IX \\
\hline - & - & & - & 55.401 & Si IX \\
\hline 0.73 & $0.89(12)$ & 0.83 & $0.99(10)$ & 50.524 & Si X \\
\hline 0.73 & $0.78(10)$ & 0.83 & $0.91(11)$ & 50.691 & Si $X$ \\
\hline 0.40 & $0.51(7)$ & 0.40 & $0.43(6)$ & 43.763 & Si XI \\
\hline 0.33 & $0.68(11)$ & 0.37 & $0.34(7)$ & 52.296 & Si XI \\
\hline 0.23 & $0.31(6)$ & 0.20 & $0.19(5)$ & 44.020 & Si XII \\
\hline 0.22 & & 0.18 & & 44.050 & $\operatorname{Mg} X$ \\
\hline 0.47 & $0.52(7)$ & 0.41 & $0.41(5)$ & 44.165 & Si XII \\
\hline 51.1 & $51.8(25)$ & 80.6 & 71.7 (29) & 171.075 & Fe IX \\
\hline 39.8 & $50.3(43)$ & 52.7 & $52.2(43)$ & 174.534 & $4 \mathrm{Fe} X$ \\
\hline 0.16 & $0.68(11)$ & 0.12 & $0.30(6)$ & 76.117 & Fe XIII \\
\hline 0.12 & & 0.11 & & 76.152 & Fe XIV \\
\hline 0.45 & $1.01(11)$ & 0.40 & $0.40(7)$ & 76.022 & Fe XIV \\
\hline 0.25 & $0.14(5)$ & 0.10 & $0.15(4)$ & 15.013 & Fe XVII \\
\hline 0.15 & $0.13(4)$ & 0.06 & $0.08(4)$ & 16.775 & Fe XVII \\
\hline 0.18 & $0.12(3)$ & 0.07 & $0.11(4)$ & 17.051 & Fe XVII \\
\hline 0.14 & 0.19 (4) & 0.06 & $0.06(3)$ & 17.100 & Fe XVII \\
\hline 6.55 & $6.05(44)$ & 5.85 & $6.54(46)$ & 148.402 & Ni XI \\
\hline 2.61 & $5.40(44)$ & 2.25 & $3.66(34)$ & 152.153 & 3 Ni XII \\
\hline 1.43 & $2.36(28)$ & 1.32 & $1.03(15)$ & 154.175 & Ni XII \\
\hline 3.69 & $3.24(35)$ & 2.30 & $1.96(23)$ & 157.730 & Ni XIII \\
\hline
\end{tabular}

${ }^{a}$ Line identification from Kelly (1987).

${ }^{b}$ Model flux in $10^{-4}$ photons $\mathrm{cm}^{-2} \mathrm{~s}^{-1}$.

${ }^{c}$ Observed flux at Earth in $10^{-4}$ photons $\mathrm{cm}^{-2} \mathrm{~s}^{-1}$ with uncertainty in parentheses in the last digits.

\section{References}

Allende Prieto, C., \& Lambert, D. L. 2001 ApJL, 556, 63

Anders, E., \& Grevesse, N. 1989, Geochim. Cosmochim. Acta, 53, 197

Drake, J. J., Laming, J. M., \& Widing, K. G. 1997, ApJ, 478, 403

Feldman, U., Mandelbaum, P., Seely, J. F., et al. 1992, ApJS, 81, 387

Flannery, B. P., \& Ayres, T. R. 1978, ApJ, 221, 175

Golub, L., Harnden Jr, F. R., Pallavicini, R., et al. 1982, ApJ, 253, 242

Grevesse, N., \& Sauval, A. J. 1998, Space Sci. Rev., 85, 161

Grevesse, N., \& Sauval, A. J. 1999, A\&A, 347, 348

Güdel, M., Audard, M., Mewe, R., et al. 2003, in Stellar Coronae in the Chandra and XMM-Newton Era, ed. F. Favata, \& J. J. Drake, ASP Conf. Ser., in press

Güdel, M., Guinan, E. F., Mewe, R., et al. 1997a, ApJ, 479, 416

Güdel, M., Guinan, E. F., \& Skinner, S. L. 1997b, ApJ, 483, 947

Hallam, K. L., Altner, B., \& Endal, A. S. 1991, ApJ, 372, 610

Kaastra, J. S., Mewe, R., \& Nieuwenhuijzen, H. 1996a, in UV and X-ray Spectroscopy of Astrophysical and Laboratory Plasmas, ed. K. Yamashita, \& T. Watanabe (Universal Academy Press, Inc., Tokyo), 411 (SPEx)

Kaastra, J., Mewe, R., Liedahl, D. A., et al. 1996b, A\&A, 314, 547

Kelly, R. L. 1987, Atomic and Ionic Spectrum lines below 2000 Angstroms: Hydrogen through Krypton, J. Phys. Chem. Ref. Data 16, suppl. 1

Meer, R. L. J., van der, Mendez, M., Kaastra, J. S., et al. 2003, in preparation

Mewe, R., Drake, S. A., Kaastra, J. S., et al. 1998a, A\&A, 339, 545

Mewe, R., Gronenschild E. H. B. M., \& van den Oord, G. H. J. 1985, A\&ASS, 62, 197 (MEKAL)

Mewe, R., Güdel, M., Favata, F., \& Kaastra, J. S. 1998b, A\&A, 340, 221

Mewe, R., Kaastra, J. S., \& Liedahl, D. A. 1995a, Legacy, 6, 16 (MEKAL)

Mewe, R., Kaastra, J. S., Schrijver, C. J., et al. 1995b, A\&A, 296, 477

Ness, J.-U., Schmitt, J. H. M. M., Burwitz, V., et al. 2002, A\&A, 394, 911

Pallavicini, R., Golub, L., Vaiana, G. S., et al. 1981, ApJ, 248, 279

Porquet, D., Mewe, R., Dubau, J., Raassen, A. J. J., \& Kaastra, J. S. 2001, A\&A, 376, 1113

Saar, S. H., \& Osten, R. A. 1997, MNRAS, 284, 803

Schmitt, J. H. M. M. 1997, A\&A, 318, 215

Schmitt, J. H. M. M. 1998, in Cool Stars, Stellar Systems and the Sun, ed. R. Donahue, \& J. A. Bookbinder, ASP Conf. Ser., 154, 463

Smith, G., Edvardsson, B., \& Frisk, U. 1986, A\&A, 165, 126 